\title{
Novel approach towards colour imaging using a scanning laser ophthalmoscope
}

\author{
A Manivannan, J N P Kirkpatrick, P F Sharp, J V Forrester
}

Department of

Bio-Medical Physics and Bio-Engineering, University of

Aberdeen, Aberdeen

A Manivannan

P F Sharp

Department of Ophthalmology, University of Aberdeen, Aberdeen J N P Kirkpatrick J V Forrester

Correspondence to: Dr A Manivannan,

Department of Bio-Medical Physics and Bio-Engineering, University of Aberdeen, Aberdeen AB25 2ZD.

Accepted for publication 3 November 1997

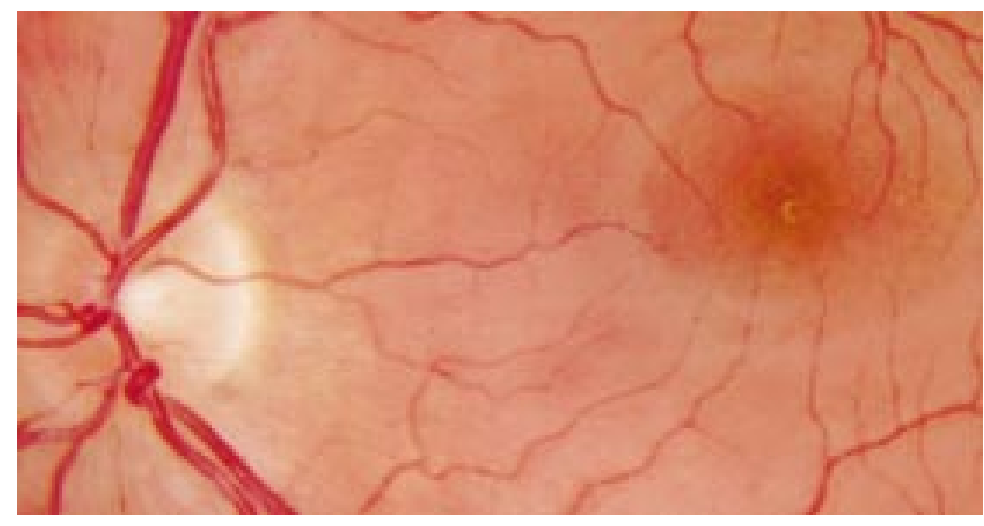

Figure 1 The normal fundus photograph of a healthy 30 year old male volunteer.

\begin{abstract}
Aims-Conventional fundus imaging using a fundus camera produces colour fundus pictures. The scanning laser ophthalmoscope (SLO) has the advantages of lower levels of light exposure, improved contrast, and direct digital imaging but until now has produced monochromatic images as a laser of single wavelength is used. True representation of the fundus is possible by combining images taken using blue, green, and red lasers.

Methods-A custom built SLO was used to capture blue, green, and red fundus images from suitable volunteers and patients with fundus disease. Images were corrected for eye movement and combined to form a colour image. Colour fundus photographs were taken using a fundus camera for comparison with the SLO image.

Results-The background fundus and retinal vasculature had similar appearances with the two imaging modalities. Internal limiting membrane reflections were prominent with the SLO. Identification of new vessels in the diabetic fundus was easier with the SLO than the colour fundus photographs.

Conclusion-A colour SLO offers all the advantages of the present monochromatic imaging system with the added advantage of true colour representation of the fundus.

(Br f Ophthalmol 1998;82:342-345)
\end{abstract}

presented in this paper were printed using a Mitsubishi colour dye sublimation printer.

Confocal scanning laser technology has been in routine use for imaging of the fundus in a variety of diseases for a number of years. ${ }^{1-5}$ A prototype SLO was built in Aberdeen ${ }^{6}$ and has been used to study device performance characteristics $^{7}$ and evaluate its potential in routine clinical diagnosis. In particular, recent work has studied the spectral reflectance of the fundus at wavelengths ranging from $488 \mathrm{~nm}$ to $830 \mathrm{~nm}^{8}$

Colour fundus photography remains the gold standard for the documentation of fundus pathology. The SLO imaging system offers some advantages over fundus photography such as improved image contrast by the selection of appropriate wavelength for the laser, ${ }^{8}$ reduced power levels, and confocal imaging but the images are monochrome. In order to overcome this we have developed a colour SLO imaging system. Patients are imaged using three primary colour lasers and the resultant images are combined to form a true colour image. In this paper, we describe the imaging system and compare selected colour SLO images with colour fundus photographs.

\section{Methods}

Using the custom built Aberdeen SLO fundus images were captured at a resolution of $768 \times$ 512 pixels using a frame grabber in an IBM compatible PC at blue (argon, $488 \mathrm{~nm}$ ), green (coumarin dye, $547 \mathrm{~nm}$ ), and red (diode, 670 $\mathrm{nm}$ ) wavelengths. Image acquisition time was $40 \mathrm{~ms}$ per frame, but allowing for the change in wavelength the total imaging time was less than 1 minute. Raw images were registered to correct for eye movement using Visilog software (Noesis, France) running on a SUN Sparc station. The intensity of the registered images was then corrected to take into account the variation in the photodiode response at different wavelengths. These correction factors were computed from images of a uniformly reflective model eye with a fundus made of Spectralon (extremely white thermoplastic diffusely reflective from $200 \mathrm{~nm}$ to $2600 \mathrm{~nm}$ (Labsphere Inc)) captured at the three wavelengths. Corrected images were then combined to form a true colour SLO image. Images 

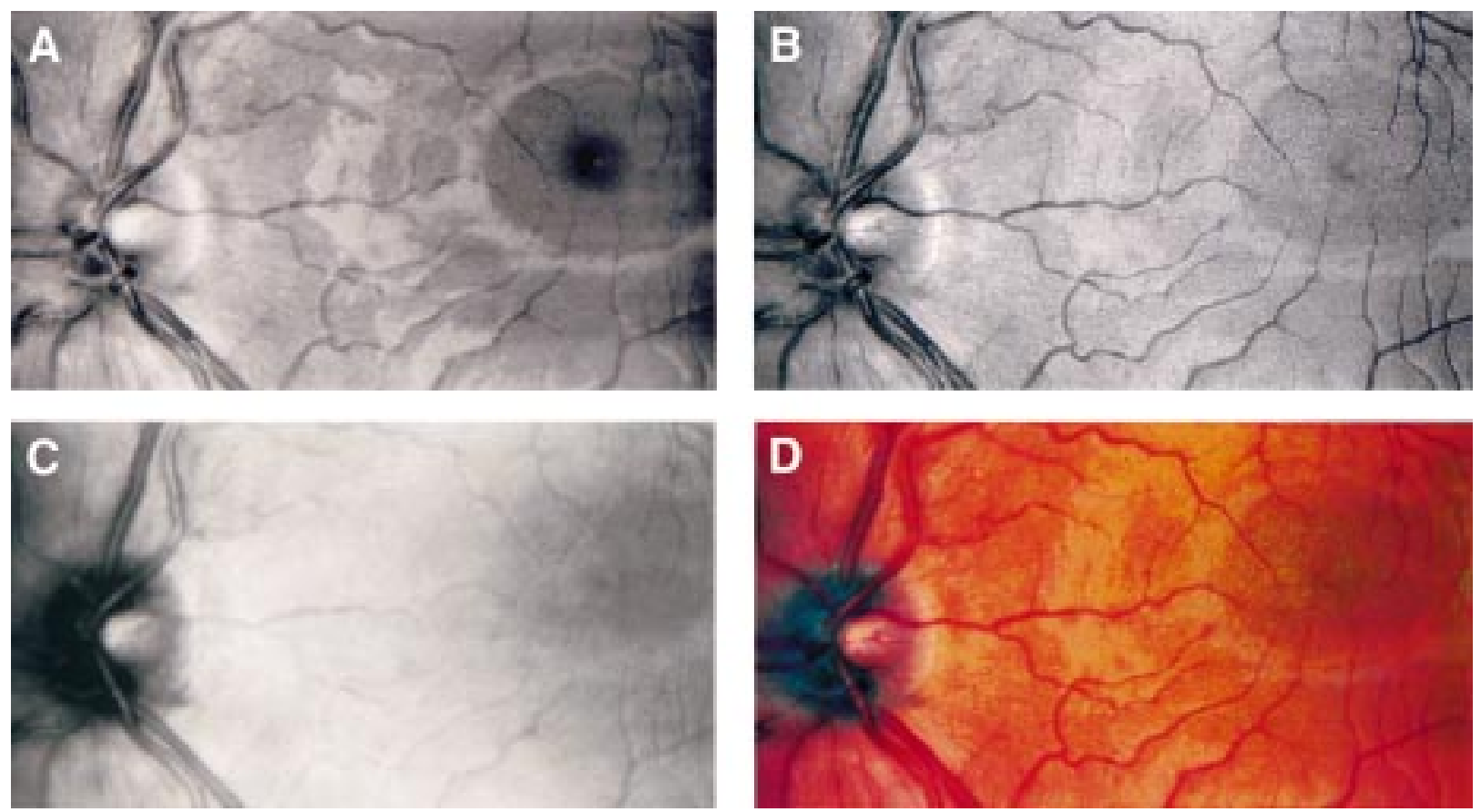

Figure 2. Monochromatic SLO images of the same volunteer taken at (A) $488 \mathrm{~nm}$ (blue), (B) $547 \mathrm{~nm}$ (green), and (C) $670 \mathrm{~nm}$ (red). The blue, green, and red images are combined to show the true colour image (D). Blue wavelengths are good for imaging the internal limiting membrane which is highly reflective at these wavelengths. Green wavelengths show the vessels at their highest contrast as blood is highly absorbed at these wavelengths. Red wavelengths can differentiate the arteries and the veins.

Suitable patients and volunteers for colour SLO imaging were selected from the eye outpatient department, Aberdeen Royal Infirmary. All subjects gave informed consent and the study was approved by the local hospital ethics committee. For comparison with the SLO colour images, colour fundus photographs were taken on the same day using a
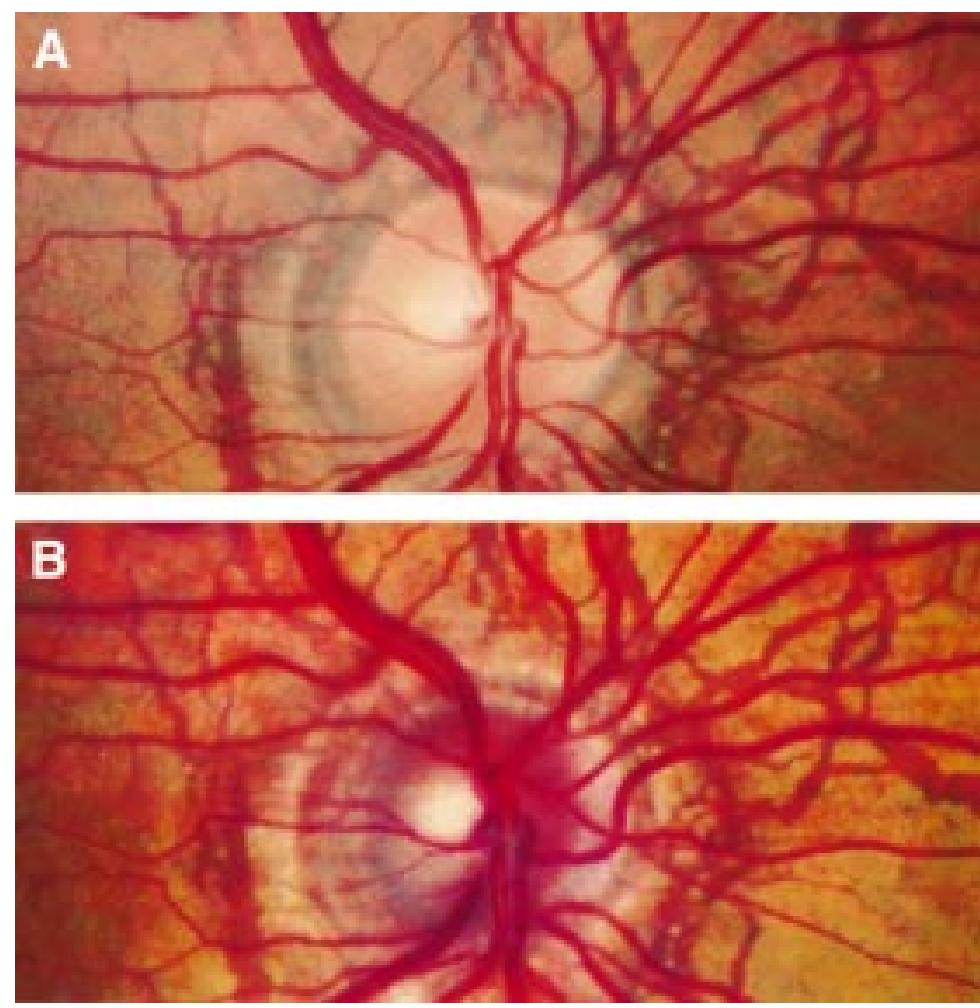

Figure 3 (A) Fundus photograph and (B) colour SLO image of a patient with angioid streaks.
Topcon TRC V50 fundus camera. Pupillary dilatation with tropicamide $0.5 \%$ and phenylephrine $2.5 \%$ was used for both photographic and SLO imaging.

\section{Results}

Photographic and colour SLO images from three patients and one normal volunteer are presented for comparison. The normal fundus of a healthy 30 year old male volunteer is presented in Figure 1. Individual registered, intensity corrected blue, green, and red monochrome SLO images of the same volunteer are shown in Figure 2. In general the background fundus and retinal vasculature have similar appearances with the two imaging modalities. Noted differences include the appearance of reflections around the macula from the internal limiting membrane present in the SLO which are not clearly apparent in the colour slide images. In addition, the optic disc in the colour SLO image has a dark appearance with a bright central optic cup. This is at variance with the uniformly bright appearance of the optic disc on the colour slide image. This feature is seen consistently in other subjects.

Figure 3 shows the colour SLO and fundus photographic images of a patient with angioid streaks. In general the resolution of fundus structure in the colour SLO image is similar to that in the fundus photograph. The colour SLO image shows that the angioid streaks have a similar brightness and hue as the retinal vessels. This is also seen in the fundus photograph. Again the neuroretinal rim of the optic disc appears darker on the colour SLO image.

Figure 4 shows a tuft of new vessels temporal to the macula in a diabetic patient imaged with both systems. In this patient, the identification of the new vessel complex is easier in the 


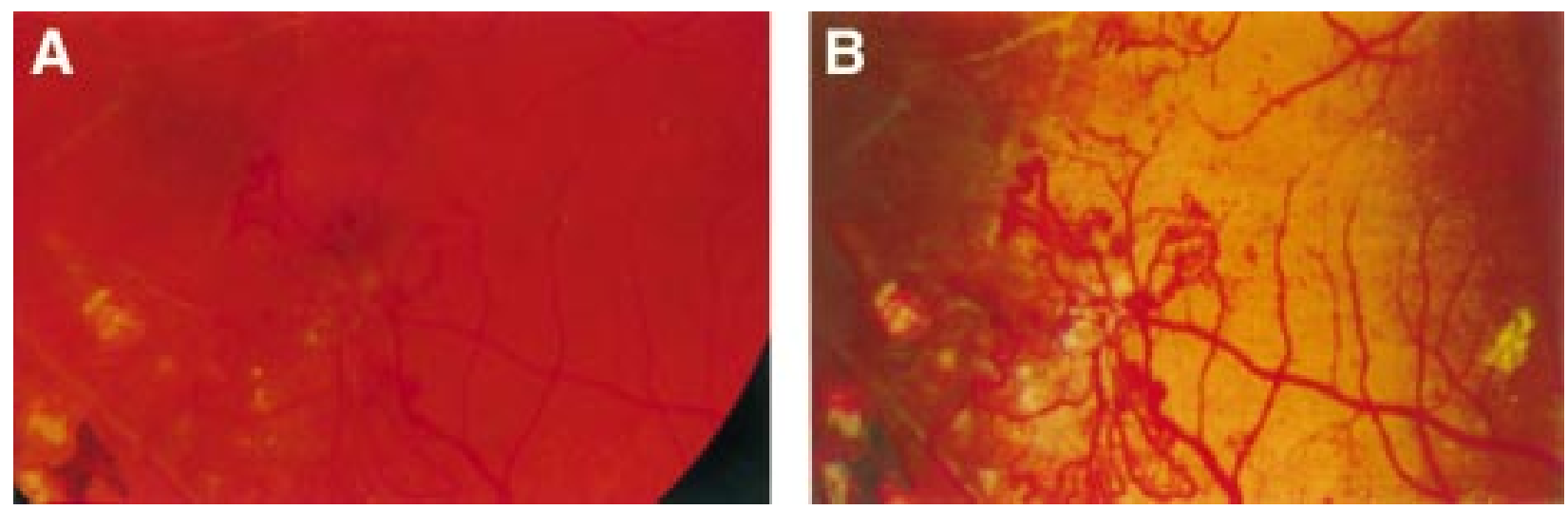

Figure 4 Tuft of new vessels temporal to the macula of a diabetic patient imaged with (A) fundus camera and (B) SLO. The new vessel complex appears with greater contrast in the colour SLO image.
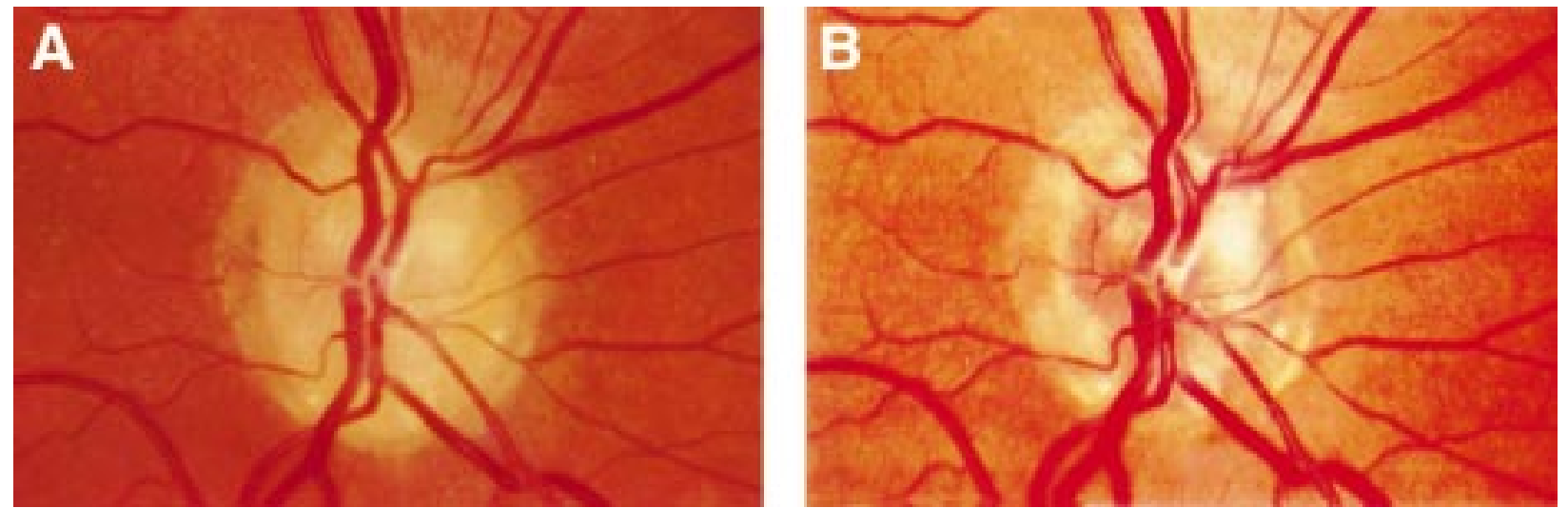

Figure 5 Optic disc drusen images using (A) fundus photography and (B) colour SLO imaging.

colour SLO image as the vessels appear with greater contrast relative to the background fundus.

Figure 5 demonstrates the appearance of optic disc drusen after colour SLO imaging and fundus photography. In both images the disc drusen appear as high intensity objects lying within the substance of the optic nerve head. However, because of the lower intensity of the neuroretinal rim in colour SLO images the contrast between disc drusen and neuroretinal rim is enhanced, making their identification easier. In both images the appearances of retinal vessels and background fundus is similar. The fine striations of the nerve fibre layer are just discernible inferior to the optic disc in both images.

\section{Discussion}

We have described a method for creation of true colour SLO images which yields high quality images that have good spatial resolution and high contrast when compared with fundus photographs. These images are of sufficient quality to act as an accurate permanent record of fundus pathology in the patients shown.

There are a number of important differences between the images produced by the colour SLO and conventional photography. The appearance of the optic disc consistently shows a darker region corresponding to the neuroretinal rim with a brighter central cup. In fundus photographs the optic disc is more uniform and of consistently higher intensity. An examination of the monochrome SLO images shows that the feature of an optic disc with a dark outer rim is present at all three wavelengths. Indeed, it is widely accepted that the optic disc appears darker on SLO imaging and this feature is enhanced by the use of confocal optics. ${ }^{2}$ It is suggested that the conventional fundus camera view of the optic disc relies on reflection of light from a large slice of the fundus which includes the highly reflective lamina cribrosa. The confocal optics of the SLO collects light from a relatively smaller depth of the optic disc and so the disc appears to have low intensity. The darker appearance of the optic disc may confer some clinical advantage as is seen in the patient with optic disc drusen (Fig 5).

Contrast of new vessels in the fundus of the diabetic patient shown in Figure 4 was notably improved in the colour SLO image. This enhanced contrast is not seen for retinal vessels in other subjects and may be due to a less than optimum exposure during fundus photography. It remains to be seen whether retinal vascular abnormalities are generally more evident in colour SLO imaging.

As the SLO method stands it is cumbersome requiring collection of three images sequentially and image processing to enable registration and intensity correction. It is envisaged that true real time colour SLO imaging is feasible with continuous illumination of the fundus by all three lasers in pulse mode to avoid overexposure. This also allows collection of pulsed 
colour signals at the detector to build up a digital colour image. The development of this method is still ongoing.

Colour SLO imaging has the obvious advantage of creating a colour view of the fundus with which clinicians are most familiar for differentiating various pathological conditions. Objects such as haemorrhages, cotton wool spots, and drusen which have a similar colour, are differentiated by features such as size, shape, edge sharpness, and texture. However, it is widely appreciated that in certain pathologies colour differences are major distinguishing factor, such as between red haemorrhages and yellow exudates. Indeed, some image processing techniques have been applied to colour fundus images specifically to take advantage of this colour information. ${ }^{9}{ }^{10}$

A colour SLO offers all the advantages of conventional monochromatic imaging system, while the problems of chromatic aberration and uncertainties of "colour developing" are avoided. Not only does it provide another imaging tool for the armamentarium of the ophthalmologist, but may also contribute significantly towards more effective computer analysis of retinal images. A number of groups have demonstrated computer algorithms for quantifying pathological features such as macular oedema, ${ }^{11}{ }^{12}$ exudates, ${ }^{13}$ and drusen. ${ }^{14}{ }^{15}$ However, since by varying the laser wavelength the contrast of these pathological features can be increased, ${ }^{816}$ then multiple wavelength imaging should offer improved accuracy for these algorithms. Present direct digital image acquisition advantages are also retained. In conclusion, a colour SLO will satisfy the requirement of a true imager for the ophthalmologists and provide a powerful tool to the physicists for the development of image processing techniques for retinal imaging. In the future one might envisage the use of any wavelength in such a system which is selected to correspond with a known reflectance peak or trough of a fundus structure under investigation.

This work was funded in part by a postdoctoral fellowship grant from Scotia Pharmaceuticals awarded to Dr Manivannan. The financial assistance of Wellcome Trust and Aberdeen Royal Hospitals NHS Trust, Scotland is gratefully acknowledged.

1 Webb RH, Hughes GW, Pomerantzeff O. Flying spot TV ophthalmoscope. Appl Opt 1980;19:1991-7.

2 Webb RH, Hughes GW, Delori FC. Confocal scanning laser ophthalmoscope. Appl Opt 1987;26:1492-9.

3 Van de Velde FJ, Jalkh AE, Katsumi O, et al. Clinical scanning laser ophthalmoscope applications: an overview In: Nasemann JE, Burk ROW, eds. Scanning laser ophthalmoscopy and tomography. Munich: Quintessenz Verlags-GmbH, 1990;35-48.

4 Plesch A, Klingbeil U, Bille J. Digital laser scanning fundus camera. Appl Opt 1987;26:1480-6.

5 Wynn-Williams GM, Crowe WE. Laser scanning: a method of retinal imaging. Australas Phys Eng Sci Med 1986;9:15362 .

6 Manivannan A, Sharp PF, Phillips RP, et al. Digital fundus imaging using a scanning laser ophthalmoscope. Physiol Meas 1993;14:43-56.

7 Manivannan A, Sharp PF, Forrester JV. Performance measurements of an infrared digital scanning laser ophthalmoscope. Physiol Meas 1994;15:317-24.

8 Sharp PF, Manivannan A. The scanning laser ophthalmoscope Phys Med Biol 1997;42:951-66.

9 Akita K, Kuga H. Digital processing of colour ocular fundus images. In: Linberg, Kaihara, eds. MEDINFO 80. Amsterdam: IFIP North-Holland Publishing Company $1980 ; 80-4$.

10 Goldbaum MH, Karz NP, Nelson MR, et al. . The discrimination of similarly coloured objects in computer images of the ocular fundus. Invest Ophthalmol Vis Sci 1990;31:61723.

11 Phillips RP, Ross PGB, Sharp PF, et al. Use of temporal information to quantify vascular leakage in fluorescein angiography of the retina. Clin Physiol Meas 1991;11(Suppl A):81-5.

12 Phillips RP, Spencer T, Ross PGB, et al.. Quantification of diabetic retinopathy by digital imaging of the fundus. Eye 991;5:130-7

13 Ward NP, Tomilson S, Taylor CJ. Image analysis of fundus photographs: the detection and measurement of exudates associated with diabetic retinopathy. Ophthalmology 1989; 96:80-6.

14 Peli E, Lahav M. Drusen measurement from fundus photographs using computer image analysis. Ophthalmology 1991;98:1128-34

15 Kirkpatrick JNP, Spencer T, Manivannan A, et al. Quantitative image analysts of macular drusen from fundus photographs and scanning laser ophthalmoscope images. Eye 1995;9:48-55.

16 Ducrey NM, Delori FC, Gragoudas ES. Monochromatic ophthalmoscopy and fundus photography:The pathologiophthalmoscopy and fundus photography:The
cal fundus. Arch Ophthalmol 1979;97:288-93. 